\title{
Complete Linkage Cluster Analysis
}

National Cancer Institute

\section{Source}

National Cancer Institute. Complete Linkage Cluster Analysis. NCI Thesaurus. Code C65185.

A clustering method in which the distance between two clusters is determined by the greatest distance between any two objects in the different clusters (furthest neighbors). 